\title{
Comparative analysis of lasing performance of oxide-confined and proton-implanted vertical-cavity surface-emitting diode lasers
}

\author{
Robert P. Sarzała · Lukasz Piskorski
}

Received: 7 July 2010 / Accepted: 20 July 2010 / Published online: 31 July 2010

(c) The Author(s) 2010. This article is published with open access at Springerlink.com

\begin{abstract}
The comprehensive optical-electrical-thermalrecombination self-consistent VCSEL model is used to compare the performance of oxide-confined (OC) and protonimplanted (PI) VCSELs and to optimise their structures. Generally index-guided (IG) OC VCSELs demonstrate lower lasing thresholds whereas both gain-guided (GG) OC and PI ones manifest much better mode selectivity. Therefore, their either low-threshold IG or mode-selective GG versions may be intentionally used for different VCSEL applications. Lasing thresholds of OC IG VCSELs have been found to be very sensitive to the exact localisation of their thin oxide apertures, which should be shifted as close as possible towards the anti-node position. PI VCSELs, on the other hand, are simpler and cheaper in their manufacturing than $\mathrm{OC}$ ones. Although lower threshold currents are manifested by PI VCSELs with very thick implanted regions, lower threshold powers are achieved in these devices with much thicker upper unaffected layer used for the radial current flow from the ring contact towards the laser axis. Paradoxically poor thermal properties of PI VCSELs enable lower lasing thresholds of slightly detuned devices. To conclude, cheaper and mode-selective PI VCSELs may be used instead of OC ones in many of their applications provided ambient temperatures and laser outputs are not too high.
\end{abstract}

\section{Introduction}

There is a principal difference between the operation of vertical-cavity surface-emitting diode lasers (VCSELs) and

R.P. Sarzała · Ł. Piskorski $(\bowtie)$

Photonics Group, Institute of Physics, Technical University

of Łódź, ul. Wólczańska 219, 90-924 Łódź, Poland

e-mail: lukasz.piskorski@p.lodz.pl

Fax: +48-42-6313639 that of edge-emitting diode lasers (EELs). The latter ones may emit radiation containing many longitudinal modes of wavelengths close to that of the maximal optical gain and only slightly different from one another. VCSELs, on the other hand, emit radiation of only one (if any) longitudinal mode of the wavelength relatively close to the above optical gain maximum but determined mostly by the cavity design. Therefore EEL cavities are always tuned to their gain spectra maxima but those of VCSELs are sometimes intentionally detuned, which gives an additional degree of freedom in VCSEL designing. As a result, designers of EELs can propose devices emitting radiation of the wavelengths solely associated with compositions and structures of their active regions. But in the case of VCSELs, it is possible to design a device emitting radiation of required wavelength, often somewhat different (practically always longer) from that connected with their active-region structure. However, it should be remembered that physics of detuned VCSELs is a little different from that of tuned ones [1] — some of its consequences will be discussed in this paper.

Currently VCSELs are generally believed to be the most suited diode lasers for their possible numerous applications including optical wireless and fibre communications, storage of information on disks using optical methods and laser printing. The above follows from their noteworthy features: inherent dynamic single-longitudinal-mode operation, low-divergence non-astigmatic circular output beams, low threshold currents of the room-temperature continuouswave operation, device geometry suitable for integration into two-dimensional laser arrays or for monolithic integration with electronic devices, compatibility with verticalstacking architecture, the ability to be modulated at very high frequencies and in situ testing possibility.

Efficiency of diode lasers depends to a considerable extent on the successful confinement of both radiation field and 
recombining carriers within the same area. In modern GaAsbased VCSELs, it is usually carried out by oxide $\mathrm{Al}_{x} \mathrm{O}_{y}$ apertures [2] created in AlAs-rich (AlGa)As layers by radial wet oxidation [3, 4]. In such oxide-confined (OC) VCSELs [5-8], the apertures may influence both the radiation field [because of much lower $(\sim 1.6)$ refractive index of $\mathrm{Al}_{x} \mathrm{O}_{y}$ than those of semiconductor layers (over 3)] and current spreading (because of high electrical resistivity of $\mathrm{Al}_{x} \mathrm{O}_{y}$ ). Therefore the oxide aperture is always funnelling the current flow, in particular from ring-shaped contacts towards the centrally located active regions, whereas the impact on radiation fields of this very thin layer depends on its position. When the aperture is localised at the anti-node position of the optical standing wave within a laser cavity (indexguided (IG) VCSELs), radial confinement of radiation fields is very large but it almost disappears-when the aperture is shifted to the analogous node position (gain-guided (GG) VCSELs) [9].

IG OC VCSELs manifest a strong index guiding connected mostly with the radial profile of the index of refraction, whereas GG ones demonstrate the gain guiding associated with the analogous radial profile of the optical gain. IG OC VCSELs exhibit usually the lowest lasing thresholds but their single-fundamental-mode (SFM) operation is in general limited to devices equipped with relatively small active regions, i.e. for relatively small output powers only, because of increasingly non-uniform current injection into larger active regions [10]. On the other hand, GG OC VCSELs are known to ensure SFM operation for distinctly larger active regions and less divergent output beams than IG ones but it is usually achieved at the expense of much higher lasing thresholds [9]. Therefore, depending on the VCSEL application under consideration, either IG or GG OC VCSEL configurations may be intentionally used.

In properly designed VCSELs, successive node and antinode positions of the optical standing wave within their distributed-Bragg-reflector (DBR) mirrors are exactly located at successive boundaries between alternative DBR layers. On the other hand, the oxide aperture is usually created within one of DBR layers, hence the desired anti-node position is then located at one of layer edges. Its very exact anti-node position is only possible, when it is placed within one of spacers [11]. Manufacturing of oxide apertures is relatively complex and troublesome. Besides, it is often difficult to create in a controlled way the exact oxide aperture of the assumed diameter [12]. Therefore it is worthwhile to consider in the case of GG VCSELs an alternative application of the proton implantation (PI) process, which is much simpler to carry out.

In the present paper, the performance of OC VCSELs will be compared and confronted with that of PI ones, especially in slightly detuned devices. Besides, for all considered VCSEL configurations, an investigation of the impact on their performance of thicknesses and localisations of the high-AlAs-mole-fraction AlGaAs layer containing the oxide aperture (in OC VCSELs) and of the proton-implanted area (in PI VCSELs) will be carried out to optimise their structures. In the analysis, the advanced $1.3-\mu \mathrm{m} \operatorname{In}(\mathrm{Ga}) \mathrm{As} / \mathrm{GaAs}$ quantum-dot (QD) GaAs-based VCSELs [13] designed for optical fibre communication will be used as a typical modern VCSEL example.

\section{The model}

Room-temperature (RT) continuous-wave (CW) performance of the 1.3- $\mu \mathrm{m} \operatorname{In}(\mathrm{Ga}) \mathrm{As} / \mathrm{GaAs}$ quantum-dot (QD) GaAs-based VCSELs is analysed with the aid of a modified version of our three-dimensional optical-electrical-thermalrecombination self-consistent VCSEL threshold model [14]. The model consists of four mutually interrelated parts:

1. the optical model describing, for successive cavity modes, their RT CW lasing thresholds, wavelengths, absorption, and intensity profiles within the laser cavity,

2. the electrical model characterising the current spreading (including carrier diffusion) between the top and the bottom contacts, the injection of carriers of both kinds into the active region and their radial out-diffusion before their recombination,

3. the thermal model characterising generation of the heat flux (non-radiative recombination, re-absorption of spontaneous radiation as well as volume and barrier Joule heating) and its spreading within the device from heat sources towards the heat sink and within the heat sink,

4. the recombination model describing recombination processes within the QD active region, i.e. furnishing information about the optical gain process being a result of the radiative bi-molecular recombination as well as about the both non-radiative mono-molecular and the Auger recombinations.

From experimental results reported by Shchekin and Deppe [15], who have found in 1.3- $\mu \mathrm{m}$ InAs QD edgeemitting diode lasers that the carrier leakage over the barriers is insignificant even at higher temperatures, we can neglect this effect and assume that all carriers injected into the active region are in fact injected into its QDs.

In this theoretical approach, all important, usually nonlinear, interactions between optical, electrical, thermal and recombination phenomena are taken into account with the aid of the self-consistent approach including: the thermal focussing, i.e. the temperature dependence of refractive indices, the self-focusing, i.e. dependence of refractive indices on carrier-concentration decreases stimulated by radiation intensity, the gain-induced wave-guiding, i.e. the 
temperature, carrier-concentration and wavelength dependences of the extinction coefficient, the temperature dependence of thermal conductivities, the temperature and carrierconcentration dependences of electrical conductivities, the temperature, carrier-concentration and wavelength dependences of optical gain and absorption coefficients and the temperature and carrier-concentration dependences of the energy gaps.

Accordingly, three-dimensional (3D) profiles of all model parameters within the whole device volume are determined not only on the basis of various chemical compositions of its structure layers but also using the self-consistent approach and taking into account current 3D profiles of the temperature, the current density, the carrier concentration and the mode radiation intensity.

OC VCSELs are known to exhibit scattering losses, which requires the vectorial optical model to be exactly determined. However we are using a somewhat simplified scalar optical model because a vectorial one needs much more time for calculation which in the case of many iteration loops used in our self-consistent algorithm is unacceptable. Besides, for standard VCSEL designs, differences between results of scalar and vectorial optical models are usually nearly insignificant [16]. Moreover, application of thin (e.g. 30-nm) oxide layers can effectively suppress scattering losses [17]. In the present paper, the standard 12-nm oxide layers are even thinner.

When any comprehensive model is prepared, its version is the result of a compromise between its exactness and usefulness. It is always possible to prepare more exact model but it is always done at the expense of increasing time necessary for its use. Therefore the most exact models are practically useless. Every time there is the problem which of the model simplifications should be chosen to decrease its computation time without losing its exactness.

Our model has been prepared following the general principle [18] that exactness of all model parts should be of the same order, because the model is as exact as its less exact part (analogously to a chain which is as strong as its weakest link). Moreover we believe that accurate taking into consideration numerous mutual and usually strongly non-linear interactions between various physical phenomena, i.e. between various optical, electrical, thermal and recombination processes, taking place within the VCSEL volume, which requires a fully self-consistent iterative approach and which is included in our model, is much more important for model accuracy than some its shortcomings of probably minor importance. Besides, the validity of our model has been confirmed experimentally [19].

There is also another comprehensive and partially selfconsistent VCSEL model known from literature [20] with some parts more exact than ours. But also in this case other than ours model simplifications (e.g. the application of effective values of some of model parameters averaged over multi-layered device regions, reduction of computational domain for some of model equations, taking into consideration only two the lowest-order cavity modes and incomplete optical-electrical-thermal-recombination self-consistency) have happened to be necessary to reduce its computation effort.

Other known VCSEL models may be found in [21], where their properties have been analysed and compared. Some of them have been claimed to be very exact, starting from the so-called first principles. However, none of them have been fully self-consistent ones, which, in the case of VCSEL modelling, is more important than slightly more exact determination of only one chosen physical phenomenon, e.g. optical gain, and omitting its interrelations with other ones.

\section{The structure}

In the nominal OC VCSEL design under consideration (Fig. 1), the concept of the QD inside the quantum well (QW) [22] has been utilised. To overcome threshold saturation problems [23, 24], the proposed QD active region is composed of five groups of three 8-nm $\operatorname{In}_{0.15} \mathrm{Ga}_{0.85} \mathrm{As}$ QWs, each containing one QD sheet layer. In each group located close to the successive anti-node positions of the optical standing wave within the cavity, QWs are separated by 32-nm GaAs barriers, mostly undoped. Besides, at both active-region edges, additional single InGaAs QWs are located containing single QD layers. The above active-region structure is similar (but not identical) to those reported by Yu et al. [25] and Chang et al. [26]. At RT, maximum of the active-region $\operatorname{In}(\mathrm{Ga}) \mathrm{As} / \mathrm{GaAs} \mathrm{QD}$ gain spectrum corresponds to about $1260 \mathrm{~nm}$ and its half-width is equal to about $30 \mathrm{~nm}$ only [1]. Therefore, to obtain the desired 1300-nm VCSEL emission, some cavity detuning with respect to the above maximum turns out to be necessary.

The $3 \lambda$ cavity is terminated by distributed-Bragg-reflector (DBR) resonator $\mathrm{Al}_{0.90} \mathrm{Ga}_{0.10} \mathrm{As} / \mathrm{GaAs}$ mirrors: the 33.5-period bottom DBR and the 23-period upper DBR. Within the first $\mathrm{Al}_{0.90} \mathrm{Ga}_{0.10} \mathrm{As}$ layer of the upper DBR from the cavity inside, the $\mathrm{Al}_{0.98} \mathrm{Ga}_{0.02} \mathrm{As}$ layer is manufactured because it is used to create in OC VCSELs the $\mathrm{Al}_{x} \mathrm{O}_{y}$ native oxide aperture. Diameters of the upper and the bottom DBRs are equal to $40 \mu \mathrm{m}$ and $80 \mu \mathrm{m}$, respectively. All structure $p$-type layers are doped with carbon whereas all $n$-type ones-with silicon. The bottom DBR is separated from the $n$-GaAs $200-\mu \mathrm{m}$ substrate by the $500-\mathrm{nm}$ buffer $n$-GaAs layer, both doped up to $5 \times 10^{18} \mathrm{~cm}^{-3}$. Both DBRs are graded ones to reduce their electrical resistance: their AlAs mole fraction is changed linearly along the 10 -nm segment of increased doping up to $5 \times 10^{18} \mathrm{~cm}^{-3}$. In their modelling, the graded DBR layers are replaced 
Fig. 1 Oxide-isolated (OI) and proton-implanted (PI) structures of the modern GaAs-based 1300-nm In(Ga)As/GaAs quantum-dot vertical-cavity surface-emitting diode laser (VCSEL) under consideration

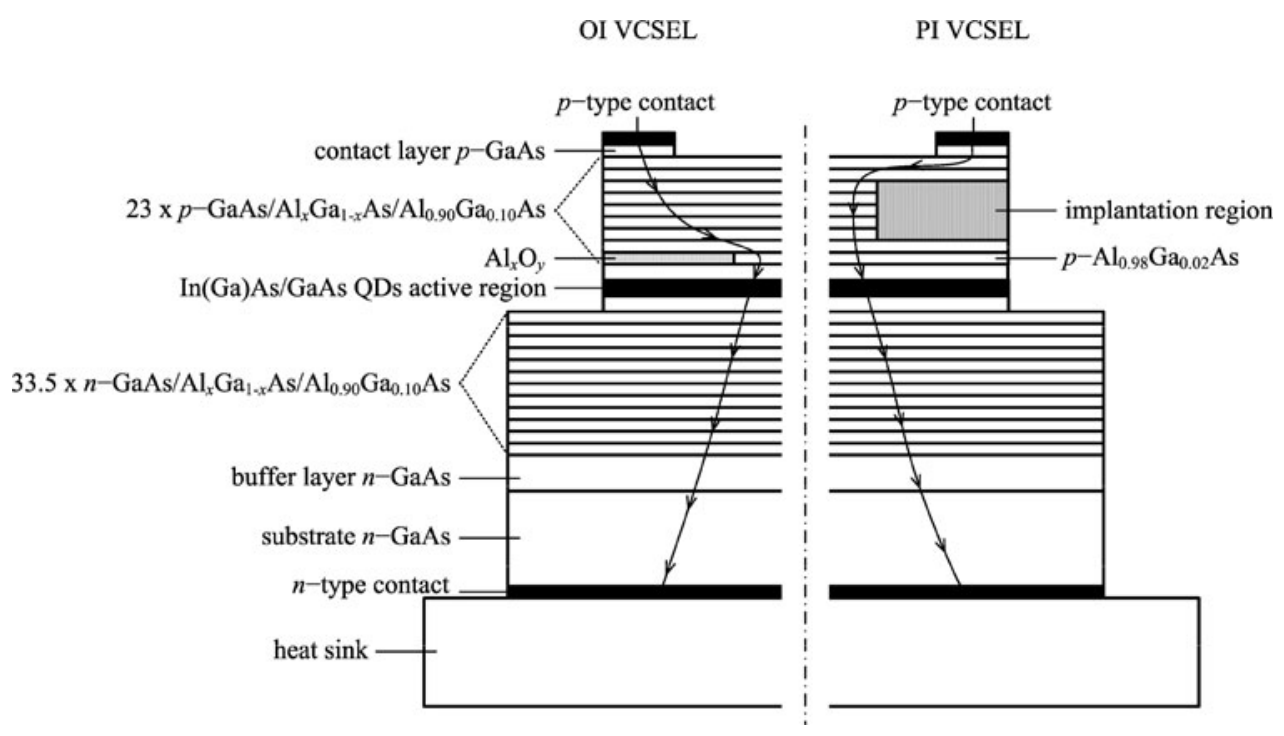

by uniform $\mathrm{Al}_{0.45} \mathrm{Ga}_{0.55}$ As layers. The GaAs layers within both DBRs are doped up to $2 \times 10^{17} \mathrm{~cm}^{-3}$, whereas the AlGaAs layers-up to $5 \times 10^{17} \mathrm{~cm}^{-3}$. The upper $p$-side ring $\mathrm{Ti}(30 \mathrm{~nm}) / \mathrm{Pt}(50 \mathrm{~nm}) / \mathrm{Au}(200 \mathrm{~nm})$ contact of internal and external diameters equal to $20 \mu \mathrm{m}$ and $40 \mu \mathrm{m}$, respectively, is separated from the upper DBR by the carbon-doped $p$-GaAs contact layer of an identical shape. The bottom $n$-side $\mathrm{AuGe}(50 \mathrm{~nm}) / \mathrm{Ni}(20 \mathrm{~nm}) / \mathrm{Au}(350 \mathrm{~nm})$ contact is deposited on the whole bottom substrate layer. The laser is attached by the $5-\mu \mathrm{m}$ indium solder to the $5-\mathrm{mm}$ diameter cylindrical copper heat sink. To funnel the current flow from the upper ring contact to the centrally located active region, the oxide apertures (in OC VCSELs) or the proton implantation (in PI VCSELs) are used.

\section{The results}

\subsection{Oxide-confined VCSELs}

Successive node and anti-node positions of the optical standing wave within a VCSEL DBR mirror are located at successive boundaries between alternative DBR layers. In the OC VCSEL design under consideration, a nominal oxide aperture is created by the selective radial oxidation of the 12-nm $\mathrm{Al}_{0.98} \mathrm{Ga}_{0.02} \mathrm{As}$ layer manufactured within the first 99-nm $\mathrm{Al}_{0.90} \mathrm{Ga}_{0.10}$ As layer of the upper DBR from the cavity. Hence the exact anti-node position corresponds to the edge of the oxide layer. Therefore it is worthwhile to examine sensitivity of OC VCSELs to some shifting and/or thickening of their oxide apertures. Figure 2 shows a possibility of optimising thickness and localisation of the oxide aperture in IG OC VCSELs under consideration in order to decrease their lasing thresholds. As one can see, apertures centrally localised within the first $\mathrm{Al}_{0.90} \mathrm{Ga}_{0.10}$ As layer and

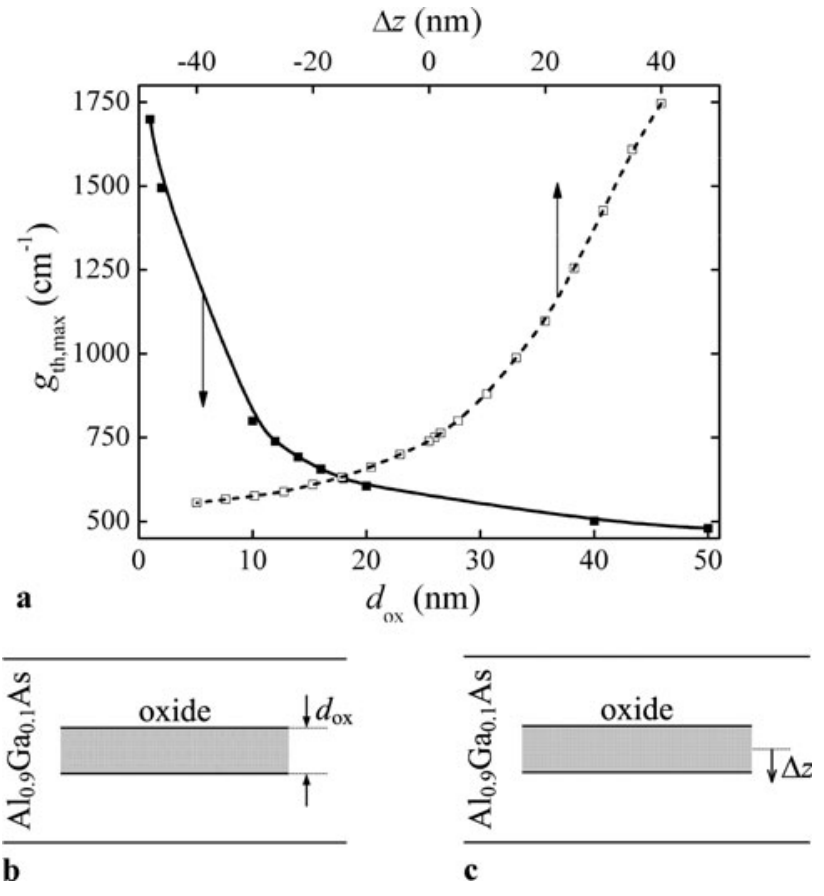

Fig. 2 The maximal RT CW threshold optical gain $g_{\text {th,max }}$ of the standard $7-\mu \mathrm{m}$ diameter $3 \lambda$-cavity IG OC QD VCSEL versus either the thickness $d_{\mathrm{OX}}$ of its oxide aperture localised in the very centre of the first 99-nm $\mathrm{Al}_{0.9} \mathrm{Ga}_{0.1}$ As layer of the upper DBR from the laser cavity or the distance $\Delta z$ from this centre of the standard 12-nm oxide aperture (negative numbers corresponds to aperture shifting towards the cavity). $d_{\mathrm{OX}}$ and $\Delta z$ are defined in $\mathbf{b}$ and $\mathbf{c}$, respectively

much thicker than those usually used in these devices would seemingly enable a drastic threshold reduction. However, it should be remembered, that a substantial increase in the thickness of the oxide layer leads to a drastic increase in scattering losses [17]. A threshold reduction may be accomplished with the aid of a simple shifting of a standard thin 12-nm aperture within the first $\mathrm{Al}_{0.90} \mathrm{Ga}_{0.10}$ As layer towards 
the cavity, i.e. closer to the anti-node position at its edge. From now on, such an edge oxidation configuration will be designated as the optimal one.

\subsection{Proton-implanted VCSELs}

A selective proton implantation applied to the upper DBR to funnel the current flow towards the active region is a considerably simpler technological process than radial oxidation used to create an oxide aperture. But the proton implantation cannot be started from the very DBR top because it is necessary to leave an unaffected top layer which enables a radial current flow from the ring contact towards the centrally located active region. Besides the implantation should be finished well over the active region not to destroy its structure and uniformity. Therefore, in our initial modelling, at least three $p$-side DBR periods from its very top as well as two ones the closest to the cavity are assumed not to be implanted.

In the PI VCSELs under consideration, the upper DBR is composed of $23 \mathrm{AlGaAs} / \mathrm{GaAs}$ periods. Following the above presented rules, implantation may extent from the third to the twentieth DBR periods, hence the maximal number of implanted periods is equal to 18 . Figure 3 presents PI VCSEL threshold currents for a reduced implanted area, i.e. versus either the number of the first implanted DBR period (when all periods over it up to the twentieth are implanted) or the last implanted period (when all periods below it from the third one are implanted). As expected, the lowest threshold current has been determined for the thickest assumed implantation area, i.e. for implantation carried out from the third to the twentieth DBR periods. Any reduction of the thickness of this implantation area is followed by a distinct increase in the lasing threshold current. Therefore the possible increase in the thickness of this area beyond

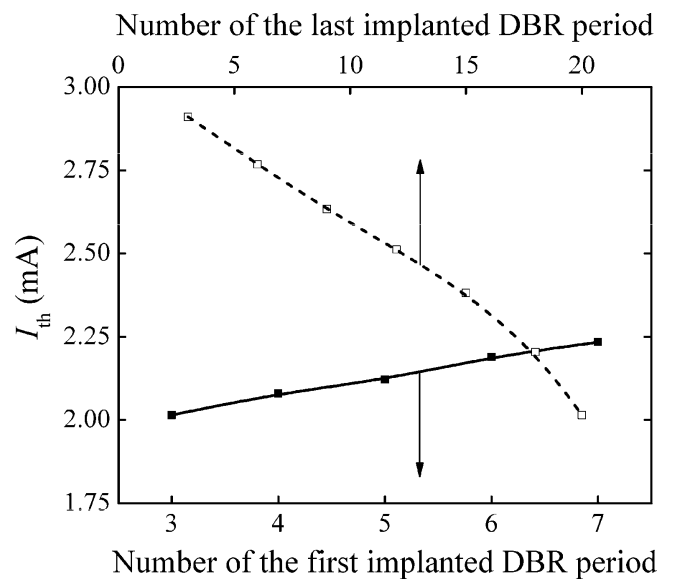

Fig. 3 The RT CW threshold current $I_{\text {th }}$ of the $7-\mu \mathrm{m}$ diameter $3 \lambda$-cavity PI VCSEL versus the number of either the first or the last implanted DBR periods (counted from the cavity) of the upper 23-period DBR the assumed limit from the $3^{\text {rd }}$ to $20^{\text {th }}$ DBR periods may be followed by a further threshold reduction. But it should be remembered, that too close approaching the active area by the implantation region may cause some increase in the non-radiative recombination. Besides a possible narrowing of the top radial-current-flow layer may cause a considerable increase in the electrical series device resistance and additional heat generation. Nevertheless, it has been confirmed by our simulation, that some shifting down of the upper implantation boundary is followed not only by some increase in the threshold current but surprisingly also by a considerable reduction of the threshold power (Fig. 4) because of a dramatic decrease in the series device electrical resistance. Therefore the optimal implantation area for the reduction of both the threshold current and the threshold power has been found to be extended from the third to the twelfth DBR periods (counted from the cavity). So, as compared with the above structure ensuring the lowest threshold current, the optimal implantation leaves much thicker top current-spreading layer to reduce device heating and electrical series resistance at the cost of some threshold increase.

\subsection{Oxidation-confined versus proton-implanted VCSELs}

Let us compare the RT CW threshold performance of 1.3- $\mu \mathrm{m}$ OC VCSELs (with the nominal 12-nm oxide apertures located both in the centre of the first $\mathrm{Al}_{0.90} \mathrm{Ga}_{0.10}$ As layer of the upper DBR from the cavity and in their optimal position) with the analogous performance of 1.3- $\mu \mathrm{m}$ PI VCSELs with both the optimal implantation and the standard one extended from the $4^{\text {th }}$ to $20^{\text {th }}$ DBR periods. Figure 5 presents radial profiles of the RT CW threshold current density determined for the 7- $\mu \mathrm{m}$ diameter active regions of QD VCSELs of various considered configurations. Unexpectedly

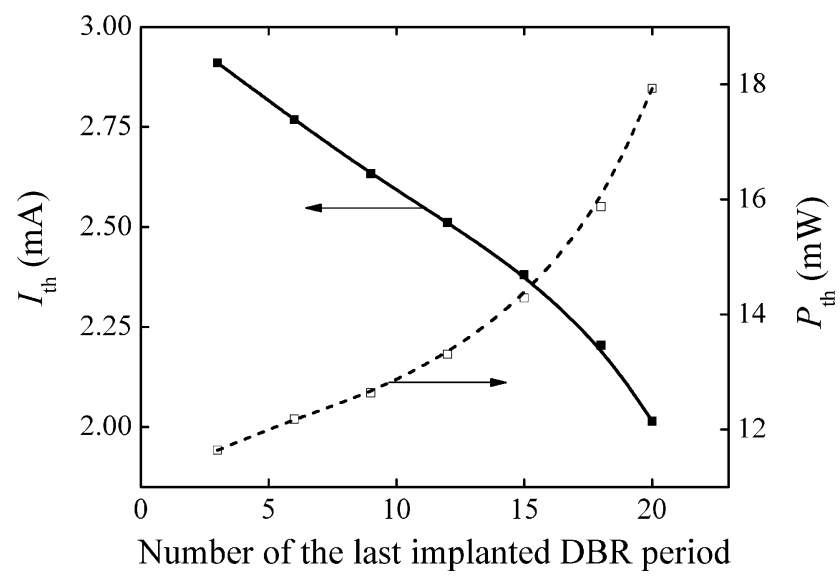

Fig. 4 The RT CW threshold current $I_{\text {th }}$ and the RT CW threshold power $P_{\text {th }}$ of the $7-\mu \mathrm{m}$ diameter $3 \lambda$-cavity PI VCSEL versus the number of the last implanted DBR period (counted from the cavity) of the upper 23-period DBR 
Table 1 RT CW threshold parameters of GaAs-based 1.3- $\mu \mathrm{m}$ VCSELs: $r_{\mathrm{A}}$ - the active-region radius, $I_{\text {th }}$ - threshold current, $U_{\text {th }}$-threshold voltage,

$T_{\mathrm{A}, \max }$ - maximal active-region temperature, $T_{\max }$-maximal laser temperature,

$n_{\text {th, } \max }$ - maximal active-region threshold carrier concentration, $g_{\text {th, } \max }$-maximal active-region optical gain, $\lambda$-wavelength of emitted radiation

\begin{tabular}{|c|c|c|c|c|c|c|c|c|}
\hline $\begin{array}{l}r_{\mathrm{A}} \\
(\mu \mathrm{m})\end{array}$ & $\begin{array}{l}I_{\text {th }} \\
(\mathrm{mA})\end{array}$ & $\begin{array}{l}U_{\text {th }} \\
(\mathrm{V})\end{array}$ & $\begin{array}{l}T_{\mathrm{A}, \max } \\
(\mathrm{K})\end{array}$ & $\begin{array}{l}T_{\max } \\
(\mathrm{K})\end{array}$ & $\begin{array}{l}n_{\text {th, } \max } \\
\left(10^{24} \mathrm{~m}^{-3}\right)\end{array}$ & $\begin{array}{l}g_{\text {th, } \max } \\
\left(\mathrm{cm}^{-1}\right)\end{array}$ & $\begin{array}{l}\lambda \\
(\mathrm{nm})\end{array}$ & mode \\
\hline \multicolumn{9}{|c|}{ optimal oxidation } \\
\hline 1.5 & 1.75 & 4.8 & 319.9 & 320.0 & 1.78 & 1002 & 1285.04 & $\mathrm{LP}_{01}$ \\
\hline 3.5 & 2.13 & 2.8 & 308.4 & 308.4 & 0.90 & 465 & 1285.81 & $\mathrm{LP}_{01}$ \\
\hline 7.0 & 4.31 & 2.8 & 319.9 & 320.0 & 0.71 & 487 & 1287.02 & $\mathrm{LP}_{11}$ \\
\hline \multicolumn{9}{|c|}{ central oxidation } \\
\hline 1.5 & 2.41 & 6.4 & 342.4 & 343.0 & 2.11 & 1501 & 1287.40 & $\mathrm{LP}_{01}$ \\
\hline 3.5 & 2.50 & 3.2 & 313.3 & 313.3 & 0.99 & 548 & 1286.38 & $\mathrm{LP}_{01}$ \\
\hline 7.0 & 4.49 & 2.9 & 321.9 & 322.0 & 0.72 & 514 & 1287.62 & $\mathrm{LP}_{01}$ \\
\hline \multicolumn{9}{|c|}{ standard implantation } \\
\hline 1.5 & 1.37 & 14.3 & 344.7 & 362.3 & 1.31 & 1172 & 1288.99 & $\mathrm{LP}_{01}$ \\
\hline 3.5 & 2.08 & 9.1 & 336.6 & 341.6 & 0.91 & 792 & 1288.66 & $\mathrm{LP}_{01}$ \\
\hline 7.0 & 3.77 & 7.3 & 351.8 & 354.0 & 0.60 & 504 & 1290.26 & $\mathrm{LP}_{01}$ \\
\hline \multicolumn{9}{|c|}{ optimal implantation } \\
\hline 1.5 & 1.57 & 9.0 & 334.7 & 343.4 & 1.57 & 1131 & 1298.99 & $\mathrm{LP}_{01}$ \\
\hline 3.5 & 2.51 & 5.3 & 325.9 & 327.0 & 1.05 & 741 & 1287.70 & $\mathrm{LP}_{01}$ \\
\hline 7.0 & 4.12 & 4.0 & 329.2 & 329.7 & 0.65 & 501 & 1288.25 & $\mathrm{LP}_{01}$ \\
\hline
\end{tabular}

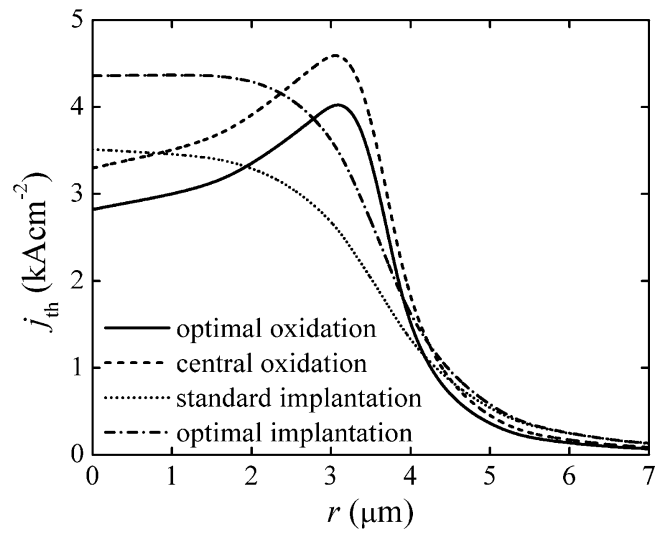

Fig. 5 Radial profiles of the RT CW threshold current density $j_{\text {th }}$ determined for 7- $\mu \mathrm{m}$ diameter QD VCSELs of various considered configurations

threshold current densities of PI VCSELs are very similar to those of OC ones, which has been found to follow from the cavity detuning and will be explained later. As expected, both optimal current-confinement mechanisms lead to lower threshold current densities. But somewhat surprisingly current-confinement mechanism in PI VCSELs has been found to be very efficient in creation of bell-like current profiles (Fig. 5) leading to an easy excitation of the desired similarly shaped fundamental $\mathrm{LP}_{01}$ mode. In the case of OC VCSELs, on the other hand, current injection into the active-region centre is distinctly lower than that close to its perimeter (cf. [10]). Then the overlapping of the local optical gain and the bell-like $\mathrm{LP}_{01}$ mode intensity profile is much worse which may be overcome to reach

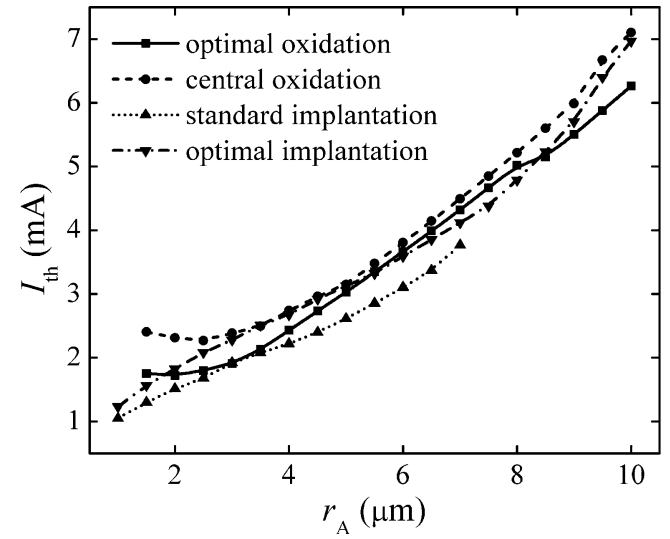

Fig. 6 RT CW threshold current $I_{\text {th }}$ of QD VCSELs equipped with various current-confinement mechanisms versus radii $r_{\mathrm{A}}$ of their active regions

the lasing threshold by an increase in the operation current only.

RT CW threshold currents of the above VCSELs are shown in Fig. 6 as a function of radii of their active regions. Some other RT CW threshold parameters are listed for all four considered VCSEL configurations and three active region radii in Table 1. For hypothetical perfect confinements of carriers and radiation field within the same area and identical heat generations, all curves plotted in Fig. 6 should be identical. Therefore any difference between them follows from some differences in the above confinements and different thermal properties. For all VCSEL configurations, threshold currents increase superlinearly with the active-region radius $r_{\mathrm{A}}$, which may be partly explained by 


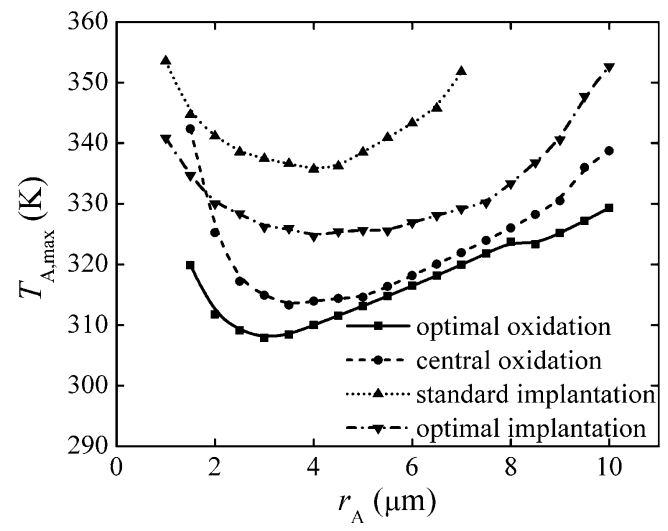

Fig. 7 The maximal active-region temperature $T_{\mathrm{A}, \max }$ determined for the RT CW threshold operation of QD VCSELs equipped with various current-confinement mechanisms versus radii $r_{\mathrm{A}}$ of their active regions

an increase in the active-area size proportional to $r_{\mathrm{A}}^{2}$. Only for very small active regions of OC VCSELs some threshold increase may be seen with the decrease in $r_{\mathrm{A}}$. As expected, an optimal oxidation ensures lower thresholds, whereas the impact of an optimal implantation is ambiguous. It follows from different optimisation goals for both VCSEL configurations: the optimal OC VCSEL has been found for reduction of the threshold current only whereas analogous optimal PI VCSEL has been assumed to keep reasonably low both the threshold current and the threshold power.

Some of PI VCSELs demonstrate in Fig. 6 and Table 1 lower lasing thresholds than analogous OC ones. This effect partly follows from the desired bell-like current injection into PI active regions (Fig. 5). But anyway it is a rather unexpected result, if a lack of radial index guiding in these devices is taken into account. The above effect results from VCSEL detuning. Then an increase in the active-region temperature is necessary to shift the relatively narrow QD gain spectrum with its maximum at $1260 \mathrm{~nm}$ towards longer wavelengths leading to higher optical gain for the desired $1300-n m$ radiation. PI VCSELs manifest very poor thermal properties, which paradoxically turns out to be very profitable in detuned QD VCSELs because their higher activeregion temperatures (cf. Table 1) are reached in a natural way, i.e. without an additional increase in the operation current as it takes place in OC VCSELs.

For all VCSEL configurations considered, Fig. 7 presents maximal active-region temperatures versus the radius of the active region. As expected, both PI VCSELs demonstrate distinctly higher temperatures than oxidised ones. The above behaviour follows from much higher series electrical resistances of PI VCSELs, being the result of a radial current flow through a relatively thin upper layer from the upper ring contact towards the device axis and, afterwards, its further axial flow through a relatively narrow area as compared with that of OC VCSELs (cf. Fig. 1). For all VCSEL designs,

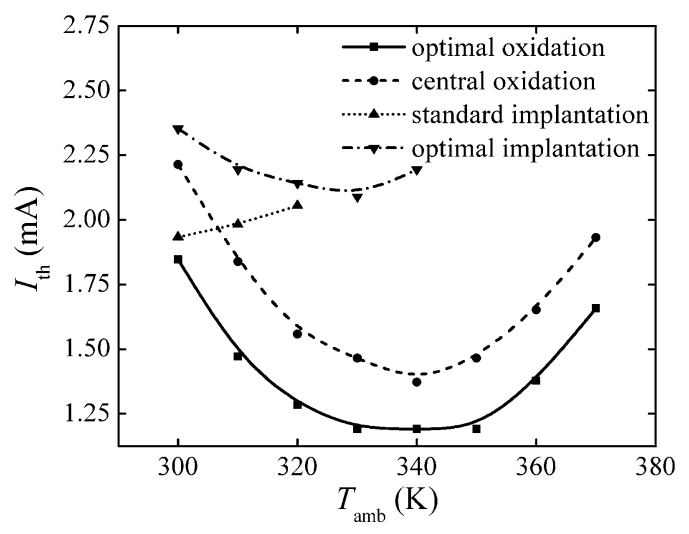

Fig. 8 Temperature dependence of the threshold current $I_{\text {th }}$ of 7- $\mu \mathrm{m}$ diameter QD VCSELs of considered configurations. $T_{\mathrm{amb}}-$ ambient temperature. PI VCSELs stop lasing beyond the indicated area

devices with active-region diameters $2 r_{\mathrm{A}}$ equal to about 6$10 \mu \mathrm{m}$ exhibit the lowest active-region temperatures.

Thermal properties of considered QD VCSELs are compared in Fig. 8. As one can see, both OC VCSELs have been found to be distinctly detuned for their RT threshold operation. Their perfect threshold tuning between the fundamental cavity mode and the active-region gain spectrum has been reached at the ambient temperature of about $340 \mathrm{~K}$, for which their threshold currents are minimal and much lower than those of PI VCSELs. However, for over-threshold lasing operation, for which the active-region temperature may be distinctly higher than that of the ambient, the above intentional detuning will play a profitable role. Besides, because of much worst thermal properties of considered PI VCSELs associated with their much higher series electrical resistances, their $\mathrm{CW}$ lasing operation is limited to ambient temperatures not higher than $340 \mathrm{~K}$ (for the optimal implantation) and only $320 \mathrm{~K}$ (for the standard one).

\section{Conclusions}

VCSEL applications often require emission of radiation of wavelengths somewhat different from that corresponding to a maximum of their gain spectrum. Then the cavity should be designed for the expected wavelength leading to some its detuning with respect to the active-region gain.

Low-threshold VCSEL operation depends to a considerable extent on the efficient confinements of its recombining carriers and optical field within the same area. OC VCSELs offer very effective methods of both the above confinements, therefore lasing thresholds of their IG version may be very low and may be reduced by shifting the oxide aperture towards the exact optical-wave anti-node position. But then their mode selectivity enabling the desired single-fundamental-mode (SFM) operation is limited to devices equipped with relatively small active regions, i.e. to 
relatively low outputs. This problem may be overcome by a reduction or even a removing of the optical-field confinement (GG OC VCSELs), which considerably improves the mode selectivity but at the expense of a dramatic increase of the threshold.

Similar GG VCSELs with only current-confinement may be produced in a much simpler way with the aid of proton implantation. The best SFM performance of such PI VCSELs may be achieved for their quite thick upper DBR areas not affected by implantation for which the current injection into the active region is similar to the desired belllike shape. Then the desired single-fundamental-mode operation is achieved for wider operation-current range than in the case of OC VCSELs. Paradoxically the poor thermal properties of PI VCSELs, which follow from their high series electrical resistances, enable reaching lower RT lasing thresholds of slightly detuned devices because their higher active-region temperatures may be reached without an additional increase in the operation current.

Generally, OC VCSELs usually exhibit lower lasing thresholds but cheaper PI VCSELs of somewhat higher thresholds enable reaching desired single-fundamentalmode operation for wider operation-current range. Therefore PI VCSEL may be surprisingly used instead of OC ones in many applications, provided ambient temperatures and laser outputs are not too high.

Acknowledgements The authors would like to acknowledge support from the grant for the Poland-Singapore scientific collaboration No SINGAPUR/19/2006 and from the Polish Ministry of Science and Higher Education (MNiSzW), grants No N N515 417635 and No N N515363736.

Open Access This article is distributed under the terms of the Creative Commons Attribution Noncommercial License which permits any noncommercial use, distribution, and reproduction in any medium, provided the original author(s) and source are credited.

\section{References}

1. Ł. Piskorski, R.P. Sarzała, M. Wasiak, W. Nakwaski, Opt. Commun. 281, 3163 (2008)

2. W. Nakwaski, M. Wasiak, P. Maćkowiak, W. Bedyk, M. Osiński, A. Passaseo, V. Tasco, M.T. Todaro, M. De Vittorio, R. Joray, J.X. Chen, R.P. Stanley, A. Fiore, Semicond. Sci. Technol. 19, 333 (2004)

3. J.M. Dallesasse, N. Holonyak Jr., A.R. Sugg, T.A. Richard, N. Elzein, Appl. Phys. Lett. 57, 2844 (1990)
4. V.M. Ustinov, A.E. Zhukov, N.A. Maleev, A.R. Kovsh, S.S. Mikhrin, B.V. Volovik, Yu.G. Musikhin, Yu.M. Shernyakov, M.V. Maximov, A.F. Tsatsul'nikov, N.N. Ledentsov, Zh.I. Alferov, J.A. Lott, D. Bimberg, J. Cryst. Growth 227-228, 1155 (2001)

5. D.L. Huffaker, L.A. Graham, H. Deng, D.G. Deppe, IEEE Photon. Technol. Lett. 8, 974 (1996)

6. D.L. Huffaker, O. Baklenov, L.A. Graham, B.G. Streetman, D.G. Deppe, Appl. Phys. Lett. 70, 2356 (1997)

7. Z. Zou, D.L. Huffaker, S. Csutak, D.G. Deppe, Appl. Phys. Lett. 75, $22(1999)$

8. J.A. Lott, N.N. Ledentsov, V.M. Ustinov, N.A. Maleev, A.E. Zhukov, A.R. Kovsh, M.V. Maximov, B.V. Volovik, Zh.I. Alferov, D. Bimberg, Electron. Lett. 36, 1384 (2000)

9. R.P. Sarzała, W. Nakwaski, J. Appl. Phys. 99, 123110 (2006)

10. R.P. Sarzała, IEEE J. Quantum Electron. 40, 629 (2004)

11. A.E. Bond, P.D. Dapkus, J.D. O'Brien, IEEE Photon. Technol. Lett. 10, 1362 (1998)

12. M. Osiński, T. Svimonishvili, G.A. Smolyakov, V.A. Smagley, P. Maćkowiak, W. Nakwaski, IEEE Photon. Technol. Lett. 13, 687 (2001)

13. N.N. Ledentsov, M. Grundmann, F. Heinrichsdorff, D. Bimberg, V.M. Ustinov, A.E. Zhukov, M.V. Maximov, Zh.I. Alferov, J.A. Lott, IEEE J Select. Topics Quantum Electron. 6, 439 (2000)

14. R.P. Sarzała, W. Nakwaski, J. Phys. Condens. Matter 16, S3121 (2004)

15. O.B. Shchekin, D.G. Deppe, IEEE Photon. Technol. Lett. 14, 1231 (2002)

16. T. Czyszanowski, W. Nakwaski, IEEE J. Quantum Electron. 41, 399 (2007)

17. B.J. Thibeault, E.R. Hegblom, P.D. Floyd, R. Naone, Y. Akulova, L.A. Coldren, IEEE Photon. Technol. Lett. 8, 593 (1996)

18. W. Nakwaski, Opto-Electron. Rev. 16, 18 (2008)

19. D.W. Xu, C.Z. Tong, S.F. Yoon, W.J. Fan, D.H. Zhang, M. Wasiak, Ł. Piskorski, K. Gutowski, R.P. Sarzała, W. Nakwaski, Semicond. Sci. Technol. 24, 055003 (2009)

20. B. Witzigmann, M. Aschwanden, V. Laino, M. Luisier, S. Odermatt, M. Streiff, A. Witzig, P. Royo, D. Vez, J. Comput. Electron. 4, 7 (2005)

21. M. Osiński, W. Nakwaski, in Vertical-Cavity Surface-Emitting Laser Devices, ed. by H. Li, K. Iga (Springer, Berlin, 2003), Chap. 5

22. G.T. Liu, A. Stintz, H. Li, K.J. Malloy, L.F. Lester, Electron. Lett. 35, 1163 (1999)

23. J.A. Lott, N.N. Ledentsov, V.M. Ustinov, V.Y. Egorov, A.E. Zhukov, P.S. Kop'ev, Zh.I. Alferov, D. Bimberg, Electron. Lett. 33, 1150 (1997)

24. M. Wasiak, M. Bugajski, E. Machowska-Podsiadło, T. Ochalski, J. Kątcki, R.P. Sarzała, P. Maćkowiak, T. Czyszanowski, W. Nakwaski, J.X. Chen, U. Oesterle, A. Fiore, M. Ilegems, Opt. Appl. 32, 291 (2002)

25. H.C. Yu, J.S. Wang, Y.K. Su, S.J. Chang, F.I. Lai, Y.H. Chang, H.C. Kuo, C.P. Sung, H.P.D. Yang, K.F. Lin, J.M. Wang, J.Y. Chi, R.S. Hsiao, S. Mikhrin, IEEE Photon. Technol. Lett. 18, 418 (2006)

26. Y.H. Chang, P.C. Peng, W.K. Tsai, G. Lin, F. Lai, R.S. Hsiao, H.P. Yang, H.C. Yu, K.F. Lin, J.Y. Chi, S.C. Wang, H.C. Kuo, IEEE Photon. Technol. Lett. 18, 847 (2006) 